Revista de Psicología Vol. 30 (2), 2012 (ISSN 0254-9247)

\title{
El análisis microgenético para el estudio del desarrollo moral: consideraciones teóricas y metodológicas
}

\author{
Alia Barrios ${ }^{1}$, Silviane Barbato ${ }^{2}$ y Angela Branco ${ }^{3}$ \\ Universidad de Brasilia
}

Se parte de la importancia del desarrollo de nueva ideas y metodologías que posibiliten el avance en la interpretación científica del desarrollo moral. La relación entre la actividad humana, los procesos de comunicación y las cuestiones de moralidad, colocan a los investigadores frente al desafío de desarrollar un abordaje metodológico del desarrollo moral a partir de un enfoque que considere los aspectos cognitivos, afectivos y socioculturales, así como el papel activo de la persona en su proceso de desarrollo. A partir de esas ideas, nuestro objetivo es proponer el análisis microgenético para el estudio del desarrollo moral, tanto desde el punto de vista teórico como metodológico, en el contexto educacional.

Palabras clave: desarrollo moral, análisis microgenético, metodología, psicología cultural.

\section{Microgenetic Analysis of Moral Development: Theoretical and Methodological Issues}

New ideas and methodologies need to be developed to advance our knowledge in the understanding of moral development. The intertwined nature of human activities, communication processes, and the numerous aspects of morality pose a challenge to researchers to construct a methodology that takes into account cognition, affect, sociocultural processes and characteristics, as well as the active role of individuals in their own development. In this paper we aim at suggesting fresh theoretical ideas and a micro genetic methodology to study moral development, particularly within educational contexts.

Key-words: moral development; micro genetic analysis; methodology; cultural psychology.

1 Magíster en Desarrollo Humano y Educación por la Universidad de La Habana-Cuba. Actualmente es Doctoranda en el Programa de Posgrado en Procesos de Desarrollo Humano y Salud del Instituto de Psicología de la Universidad de Brasilia. Dirección postal: SHCES Quadra 911 Bloco B apto. 304. Cruzeiro Novo, Brasilia-DF, Brasil, CEP: 70655-002. Correo electrónico: aliabarrios@gmail.com

2 Doctora en Psicología y Profesora del Departamento de Psicología Escolar y del Desarrollo en el Instituto de Psicología en la Universidad de Brasilia. Coordina proyectos de investigación en Procesos de Desarrollo y Cultura y Procesos Educacionales. Correo electrónico: barbato. silviane@gmail.com

3 Doctora en Psicología por la Universidad de São Paulo y post-doctora, coordina el Laboratorio de Microgénesis en las Interacciones Sociales. Correo electrónico: ambranco@terra.com.br 

El desarrollo moral ha sido, cada vez más, objeto de estudio de la psicología contemporánea en función de diversos motivos. En primer lugar, por la relevancia psicológica de la dimensión estructuradora de la moral, presente en nuestras interacciones y en las relaciones con los otros, así como con el mundo del formamos parte. El encuentro con el otro ocurre en la intensificación de los procesos de movilidad inter e intra cultural de las diferentes regiones del mundo, suscitados por movimientos locales y globales (Canclini, 2003). En segundo lugar, por la importancia del estudio y de la comprensión científica del desarrollo moral para el entendimiento del desarrollo integral del ser humano. En este estudio, enfatizamos la importancia de comprender el papel del sistema educacional en relación al desarrollo moral, cuando los niños y jóvenes de diferentes culturas expanden sus posibilidades de socialización más allá de la familia y de la comunidad donde viven, enfrentando frecuentemente situaciones asimétricas y situaciones no recíprocas con el otro o con grupos situados diferentemente (Bauman, 2001). Son varios los estudios que han defendido la necesidad de que la educación contemple el desarrollo moral para una vida con autonomía y responsabilidad por las acciones sociales (e.g. Araújo, 1996; DeVries \& Zan, 1998; La Taille, 1998; Martins \& Branco, 2001; Puig, 1998).

Los estudios revisados abarcan el tema desde varios ángulos y a partir de diferentes abordajes teóricos y metodológicos. Podemos citar, a modo de ejemplo, las investigaciones realizadas por Araujo (1996), Ardila-Rey y Killen (2001), La Taille (2001), así como Lourenço y Kahn (2000), entre otros. Algunas de esas investigaciones se centran en el estudio del papel que la educación tiene en el desarrollo moral. Otras se centran en el estudio de las concepciones que los niños tienen sobre cuestiones y conceptos relativos a la moral, tales como: reglas morales, virtudes, convenciones sociomorales y la noción de justicia. Cada uno de ellos tuvo contribuciones importantes para el estudio 
del desarrollo humano, de forma general, y para el desarrollo moral, de forma más específica.

Sin embargo, algunas investigaciones intentan aproximarse de su objeto de estudio de forma fragmentada y descontextualizada. Fragmentada, debido a que analizan las concepciones sociomorales a partir de categorías previamente establecidas, que restringen el desarrollo moral al dominio cognitivo y/o social, dejando de lado sus dimensiones situacionales y subjetivas. Descontextualizadas, una vez que preocupadas con el procedimiento estadístico de los datos colectados se aproximan a su objeto de estudio a través de instrumentos estandarizados que no consideran las interacciones y eventos sociales en que esas concepciones sociomorales son construidas de forma paulatina. La mayoría de las investigaciones estudia las concepciones sociomorales a partir de conflictos y dilemas morales que, muchas veces, no reflejan la realidad sociocultural en la cual el individuo está inmerso.

La tradición iniciada por Piaget (1932/1994) continúa dominando los estudios sobre el desarrollo moral y, específicamente, sobre las concepciones y conceptos relativos a la moral, sin embargo, defendemos en este texto la necesidad de estudios que contemplen el desarrollo moral en el contexto de la actividad humana y de los procesos comunicativos (Martins, 1995; Tappan, 1992), una vez que la moral está presente en las interacciones sociales que acontecen en los eventos concretos y cotidianos (Packer \& Richardson, 1991). La acción situada en contextos socioculturales específicos debe ser un punto central tanto para el estudio del desarrollo humano (Bruner, 1997) como para el estudio del desarrollo moral.

Martins (1995) señala la necesidad de ampliar las investigaciones sobre el tema en diversas direcciones. Además de la necesidad de estudiar las concepciones sociomorales, es fundamental incorporar su relación con las interacciones y eventos sociales, en los cuales ellas se construyen poco a poco, usando entre sus métodos y procedimientos de estudio la observación de las interacciones significativas para las mismas. 
El análisis de la comunicación es fundamental para el estudio del desarrollo moral. De acuerdo con Tappan (1992), el desarrollo del funcionamiento moral es mediatizado por el lenguaje, en forma de diferentes 'voces' o 'lenguajes' morales, provenientes del contexto sociocultural en el cual el individuo vive. Esas 'voces' y 'lenguajes' morales se originan en las interacciones del niño con otras personas.

Otros autores como Martins y Branco (2001) se apoyan en estudios y reflexiones presentados por Shweder y Much (1987), y enfatizan la necesidad de desarrollar un abordaje teórico y metodológico que trate el desarrollo moral a partir de un enfoque amplio, en el cual los aspectos cognitivos, afectivos y socioculturales sean considerados de forma integrada y sistémica, tomando en cuenta la centralidad de la persona como agente autónomo de un mundo organizado culturalmente.

De forma general, las perspectivas socioculturales resaltan la importancia del análisis de los procesos comunicativos para el estudio del desarrollo humano y, específicamente, para el estudio del desarrollo moral. Es a partir de esos argumentos que reflexionamos sobre el estudio del desarrollo moral como proceso dinámico, que tiene lugar en el contexto de las interacciones y eventos sociales específicos y significativos para el individuo. Pretendemos, de forma particular, abordar la importancia del análisis microgenético para el estudio del desarrollo de la moralidad, tanto desde el punto de vista teórico como metodológico.

\section{El estudio de la moralidad en la psicología}

En la psicología contemporánea, podemos citar diversas perspectivas teóricas que han dedicado sus esfuerzos al estudio del desarrollo de la moralidad, enfatizando diversos aspectos del mismo. Entre ellas se destacan el constructivismo, la perspectiva cognitivo desarrollista, el constructivismo social y las perspectivas socioculturales.

El estudio del desarrollo moral a partir de una perspectiva constructivista tuvo inicio con los trabajos de Piaget (1932/1994) sobre el tema y, específicamente, sobre los procesos de construcción de las reglas. La teoría de Piaget aborda el desarrollo moral como un proceso de construcción que tiene lugar en los contextos de interacción social. 
La convivencia social del niño con sus pares y con los adultos permite el establecimiento de relaciones fundamentales para el desarrollo de la moralidad. Siendo así, el desarrollo moral no es un proceso de imposición de reglas y de virtudes, sino un proceso que exige una transformación de las estructuras cognitivas y que acontece en estrecha relación con el desarrollo cognitivo y los estímulos del ambiente (Duska \& Whelan, 1994).

Piaget (1932/1994) realizó diversos estudios sobre el origen y el desarrollo de las estructuras cognitivas y del juicio moral en los primeros años de vida, analizando las actitudes verbales de los niños en relación a las reglas del juego y a la noción de justicia. El método clínico usado por Piaget (1932/1994) consistía en una entrevista con preguntas relativas a las reglas de los juegos y al nivel de consciencia sobre las mismas por parte de los niños. Las informaciones analizadas cualitativamente apuntaban como los niños observaban las reglas y el nivel de consciencia que tenían sobre las mismas, en función de su edad y de su desarrollo mental. Para Piaget (1932/1994), las innovaciones individuales y lingüísticas en relación a las reglas atendían una necesidad general o una realidad social cuando eran consideradas de acuerdo con el 'espirito del juego'. Para evaluar el sentido de justicia, Piaget (1932/1994) partía de historias sobre transgresiones que los niños podían evaluar en términos de responsabilidad objetiva o subjetiva. También podían proponer sanciones expiatorias y arbitrarias o sanciones por reciprocidad y analizar la justicia en términos de obediencia a la autoridad, de igualdad o de equidad (Lourenço, 1998).

En el método clínico de Piaget es importante pedirle al niño que justifique sus respuestas iníciales a partir de contra sugerencias presentadas por el entrevistador. Las respuestas de los niños y sus reacciones de aceptación, recusa o dudas delante de las contra sugerencias son la base para saber si el niño se encuentra en una fase de moralidad heterónoma, autónoma o de transición (Lourenço, 1998). Aunque las respuestas de los niños sean analizadas de forma cualitativa, las historias usadas por Piaget (1932/1994) parten de una visión universal de la moral y no llevan en cuenta las experiencias reales de vida vinculadas a las cuestiones 
y conflictos de carácter moral (Shweder \& Much, 1987). Por otro lado, el análisis realizado se centra en la constatación del nivel de desarrollo moral alcanzado por el niño y no en los procesos relativos a ese desarrollo.

Como señala Lourenço (1998), Piaget estaba interesado en la génesis de la moralidad, por lo no se preocupó con la estandarización de las historias, con el objetivo de analizar la heteronomía y la autonomía moral de los niños desde un punto de vista cuantitativo. Esa estandarización y análisis cuantitativo fue una preocupación de investigadores constructivistas como Kurtines y Pimm (1983), por ejemplo. Siguiendo la tradición iniciada por Piaget (1932/1994), podemos encontrar diversas perspectivas teóricas que se debruzan sobre el estudio del desarrollo moral, enfatizando diferentes aspectos estructurales y funcionales del mismo, así como el principio genético. Podemos destacar los trabajos teóricos y empíricos de Kohlberg (1984), así como los de autores de la vertiente cognitivo desarrollista (e.g. Biaggio, 1983; La Taille, 2006; Lourenço, 1998). Esa vertiente enfatiza aspectos estructurales, formales y universales de la razón. Partiendo del paralelismo entre el desarrollo cognitivo y el desarrollo moral, y la idea de Piaget de que la moral es una lógica de la acción, como la lógica es una moral del pensamiento, la perspectiva cognitivo desarrollista resalta que determinado nivel de pensamiento (pensamiento lógico formal) es una condición necesaria, pero no suficiente, para que el individuo pueda alcanzar niveles más elevados en su desarrollo moral. Las otras condiciones necesarias serían la posibilidad de asumir el punto de vista del otro y la posibilidad de incluir principios de justicia en los juicios y acciones morales.

En la perspectiva cognitivista desarrollista, el estudio de la moralidad implica asumir un conjunto de principios meta éticos que se relacionan directamente con la no neutralidad (Lourenço, 1998). No es posible estudiar el desarrollo moral sin partir de valoraciones sobre los propios valores y sin correr el riesgo de caer en el relativismo ético y cultural y en la mera descripción de las acciones morales del individuo. Lourenço (1998) defiende que el estudio científico del desarrollo moral debe asumir que la acción moral es un todo que abarca la conducta y la motivación cognitiva (fenomenismo), que es necesario 
aceptar algunos principios éticos aplicables a todas las personas y culturas (universalismo), que es necesaria la obligación de obedecer siempre semejantes principios éticos (prescriptivismo), que la acción moral debe tener siempre razones morales (cognitivismo), que los principios morales resultan más de la actividad estructuradora del sujeto que de la influencia directa del medio (constructivismo), y que la justicia es el principio moral básico y el aspecto más estructurador del raciocinio moral (orientación para la justicia).

La metodología cuantitativa utilizada por los investigadores de la vertiente cognitivo desarrollista implica el uso de diversos métodos tales como entrevistas, partiendo de dilemas morales y tests de opción múltiple. Con base en los datos obtenidos se atribuye al pensamiento moral una determinada fase moral (Kohlberg, 1984), un índice cuantitativo de desarrollo moral (Rest, 1984), o un nivel de entendimiento interpersonal (Selman, 1980). El referido análisis sigue los principios apuntados por Lourenço (1998) y señalados anteriormente en el presente trabajo. Siendo así, aspectos contextuales y socioculturales del desarrollo no son llevados en cuenta (Rogoff, 2005; Shweder \& Much, 1987).

$\mathrm{Al}$ analizar los diferentes abordajes teóricos y metodológicos del desarrollo moral, Lustosa (2005) y Martins (2000) identifican como abordajes narrativos aquellos que se preocupan con los aspectos contextuales del desarrollo y se centran en las interpretaciones de las representaciones narrativas de los individuos, en el marco de sus experiencias reales de vida vinculadas a cuestiones y conflictos de carácter moral. Entre los investigadores que se sitúan en el abordaje narrativo de la moralidad se destacan los trabajos e ideas de Gilligan (1982), Goodnow (1995), Tappan (1992), Shweder y Much (1987). Los abordajes narrativos se asocian al constructivismo social, una vez que enfatizan la importancia de considerar la diversidad de la realidad sociocultural que se manifiesta de forma concreta en la estrecha relación que existe entre la cultura y el lenguaje. De esa forma, el estudio de la narrativa permite comprender el funcionamiento del universo moral del individuo en un contexto concreto, así como sirve de auxilio a la hora de comprender las transformaciones que acontecen en términos de juicio moral. 
La ética del cuidado, entendida como el sentido de responsabilidad en relación al otro y sus necesidades fue enfocada en el trabajo de Gilligan (1982). Diferente de la ética de la justicia, tema central de la perspectiva cognitivo desarrollista, la ética del cuidado remite a la intimidad, relación e interdependencia. De acuerdo con la autora, esas cuestiones no deben ser estudiadas desde la perspectiva de un self racional y abstracto, sino de un self dialógico, emocional y sensible a los contextos locales. Siendo así, el estudio de la moralidad debe valorizar las historias de vida y narrativas personales de los individuos, que están inmersos en contextos diferentes. Otra característica importante del trabajo de esta investigadora es el abordaje de cuestiones fundamentales como las diferencias de género y papeles sociales en la vida real.

A partir de un abordaje hermenéutico, Tappan (1992), que parte del importante papel del lenguaje en la constitución y entendimiento de los procesos psicológicos y, en especial, en la constitución y en el entendimiento de la dimensión psicológica del desarrollo moral, resalta la importancia del contexto sociocultural en el cual esos procesos psicológicos se constituyen. A partir de la estrecha relación entre la cultura y el lenguaje, el autor resalta que el estudio del material lingüístico no solo permite comprender la constitución y el funcionamiento del universo moral, sino que también permite comprender la diversidad sociocultural donde ese universo se constituye y funciona.

El autor busca sus fuentes teóricas en los trabajos de Bakhtin (1981, 1993) y Vygotsky $(1934 / 1968,1960 / 1987)$ sobre la función constitutiva y mediatizada del lenguaje en los procesos psicológicos y aprovecha las intersecciones y complementos en los abordajes de los dos autores. Tanto Volosinov (1929/1995) como Vygotsky (1960/1987) resaltan el carácter mediatizado del funcionamiento psicológico, el papel del lenguaje como 'instrumento, medio o herramienta' de las funciones psicológicas y su carácter sociocultural. Para Vygotsky (1934/1968), el funcionamiento psicológico está mediado por el lenguaje, que funciona como una 'herramienta psicológica', siendo esta un fenómeno sociocultural envuelto en los procesos de la comunicación y de las relaciones sociales. El lenguaje en el dialogismo tiene origen social 
y las leyes lingüísticas son leyes sociales (Volosinov, 1929/1995). Vale la pena destacar que, aunque Tappan (1992) resalta la importancia del proceso de internalización presentado por Vygotsky (1960/1987), el autor no especifica cuál es el papel del individuo en ese proceso. Por momentos, la internalización aparece como una mera apropiación o como una simple reproducción de las voces, que son combinadas de forma singular por el individuo (Lustosa, 2005).

De acuerdo con Volosinov (1929/1995), la palabra es el medio de la consciencia, es el material semiótico de la vida interna, siendo que el término 'palabra' puede ser interpretado como el discurso, como la expresión concretizada en la interacción que dirige las formas de pensar, sentir y actuar. Las enunciaciones resultan de la interacción entre voces polifónicas presentes en un constante dialogo interno y externo. El dialogismo se define como proceso dialéctico provocado por la materialización de la expresión en el enunciado que dirigido al otro también ejerce un efecto reversible sobre la actividad mental del interlocutor que habla (circularidad), por el hecho de haber sido expuesto al otro y a su propia enunciación. Esa característica de la comunicación humana implica entrar de forma mutua en el tono del otro, sintonizarse con la sintonía del otro (Bakhtin, 1981). Las voces se forman inter e intra generacionalmente en las diferentes culturas y se concretizan en las interacciones entre determinados interlocutores en un tiempo y lugar específicos. "Una voz no está apenas compuesta por una palabra o expresión, sino que es un posicionamiento semántico, un punto de vista orientado entre otros" en una determinada situación (Emerson, 1984, p. XXXVI). Las diferentes voces con las cuales alguien interaccionó/interacciona se forman por el juego entre las fuerzas de permanencia y cambio (fuerzas centrípetas y centrífugas), son el lugar de la construcción híbrida, que resulta de estructuras históricas que se actualizan en su dependencia con los elementos de la situación sociocomunicativa, y que se concretizan en una interacción. La voz personal se concretiza en enunciados dentro de interlocuciones, resultantes de una tesitura entre la cultura y la vida en la cual "el aspecto individual-histórico (el autor, el tiempo, las circunstancias 
y la unidad moral de su vida)" (Bakhtin, 1993) se expresa en actos performáticos.

Las diferentes formas en que las voces entran en contacto definen la idea de dialogía, que es mucho más amplia que el sentido asociado al término 'diálogo' en la ciencia contemporánea (Wertsch \& Smolka, 2003), pues a partir de la noción de 'voz' los aspectos esenciales de un enunciado pueden ser entendidos como una respuesta al enunciado de otra voz (Bakthin, 1993) o incluso a la previsión del enunciado del otro.

Tomando en consideración los argumentos anteriores y el análisis de varias investigaciones realizadas por Gilligan (1982), Tappan (1992) desarrolla una serie de ideas teóricas y metodológicas importantes para la comprensión y el estudio del funcionamiento moral, contraponiéndose a los autores de la perspectiva cognitivo desarrollista. Para el autor, el funcionamiento moral está mediado por el lenguaje en la forma de diferentes 'voces morales' (o 'lenguajes morales'), provenientes del contexto sociocultural en el cual el individuo vive. Siguiendo la tradición sociocultural, el lenguaje moral aparece primero entre los individuos, como categoría interpsicológica que es internalizada para aparecer como categoría intrapsicológica. El funcionamiento moral, una vez internalizado, debe ser visto como un proceso o una función psicológica compleja que abarca dimensiones psicológicas interdependientes: cognición, emoción y acción. Siendo así, el lenguaje moral y la acción moral hacen parte de la misma función psicológica compleja, aunque las mismas estén impregnadas de significados socioculturales específicos. Las 'voces morales' abarcan el conjunto de significados, concepciones y valores que surgen en un contexto sociocultural e histórico especifico, y que son internalizados por el individuo en el contexto de los procesos de comunicación e interacción.

Además de eso, Tappan (1992) presenta la relación entre lenguaje, cultura y funcionamiento moral como la relación entre textos y contextos. De acuerdo con el autor, el dialogo entre las 'voces morales' que los individuos invocan para resolver problemas, conflictos o dilemas morales aparece representado en los textos de las entrevistas realizadas 
en investigaciones, demostrando que los seres humanos no solo son polifónicos sino que también pueden oscilar de una voz para otra a la hora de resolver problemas. Esas voces o lenguajes morales son un fenómeno sociocultural, por tanto están situadas socioculturalmente, ocupando un lugar predominante en los diferentes dominios de la vida privada y pública. Para el autor, eso significa que esas voces o lenguajes morales deben ser interpretadas a partir de lo que Bakhtin denominó de lenguaje social, o sea, un discurso característico de un determinado segmento de la sociedad que pertenece, al mismo tiempo, a un sistema social determinado más amplio. Para entender el funcionamiento moral de un individuo es necesario entender las voces culturales vehiculadas en las interlocuciones concretizadas en géneros discursivos preferenciales en un cronotopo (Bakhtin, 1981, 1993).

En el estudio del desarrollo moral dentro de esta perspectiva, el lenguaje aparece como una instancia fundamental de su constitución y como un medio de acceso a la experiencia moral del individuo. En función de eso, el estudio de la narrativa del individuo delante de situaciones y problemas morales específicos permite comprender la constitución y el funcionamiento moral de forma concreta, así como permite comprender las transformaciones que ocurren en el funcionamiento moral, una vez que el mismo no es estático ni acontece de forma abstracta. En la narrativa, esas transformaciones son inherentes a la diversidad con que la cuestión de la moralidad se manifiesta en la vida cotidiana (Martins, 2000).

Las perspectivas socioculturales que enfatizan simultáneamente la individualidad y la relación de la persona con los contextos culturales en que vive buscan extrapolar la parcialidad de los modelos teóricos que se preocupan en demostrar la 'dilución' de los individuos en los contextos sociales, y se encaminan para la superación de la oposición existente ente las perspectivas universalistas y narrativas del desarrollo moral. Autores como Rey y Martinez (1989) y Rogoff (2005) resaltan la importancia del análisis de los procesos comunicativos para el estudio del desarrollo humano, de forma general, y para el estudio del desarrollo moral, de forma más específica. Para estos autores, inspirados 
en el desarrollo de las tesis de Vygotsky, la cultura, que es constitutiva de la mente humana, debe ser comprendida como interacciones intersubjetivas y como interacciones con los artefactos culturales, simbólicos y materiales. Interacciones dinámicas, complejas y dialécticas que resaltan la importancia de la centralidad de la persona como agente autónomo de un mundo organizado culturalmente.

Rogoff (2005) no solo resalta el carácter dialógico de los procesos de desarrollo humano, sino que también enfatiza que esos procesos de desarrollo tienen lugar en el contexto de prácticas culturales específicas, en las cuales el individuo participa de forma activa y en conjunto con los otros. Siendo así, acción, comunicación y prácticas socioculturales aparecen como elementos interconectados y fundamentales para el estudio del desarrollo moral. Para la autora, el desarrollo humano acontece mientras las personas (que no son genéricas) participan en actividades socialmente organizadas y mediatizadas por el lenguaje con discursos específicos (prácticas socioculturales), en diferentes contextos sociales. Las variantes, diferencias y semejanzas de las prácticas socioculturales, de la participación de los individuos y de los discursos de un contexto para otro, deben ser estudiados y comprendidos, a partir de un programa de investigación que puede ser caracterizado a partir de las siguientes premisas: a) reconocimiento de la naturaleza holística del desarrollo humano, o sea, las personas y las actividades se constituyen mutuamente; b) observación de los procesos de desarrollo por la participación orientada en las prácticas y en los contextos culturales; y c) prioridad de las metodologías cualitativas en el estudio de los individuos en los contextos culturales. Su trayecto como investigadora conjuga el estudio etnográfico de los fenómenos y de los diferentes modelos de la psicología popular (folk psychology) existentes, utilización de material videográfico, entrevistas, observaciones, experimentación en contextos reales de vida y en laboratorio. Los trabajos en laboratorio tienen como objetivo profundizar las cuestiones que surgen en los estudios etnográficos de la vida diaria (Costa \& Lyra, 2002; Fidalgo, 2004). 
Las perspectivas narrativas y socioculturales presentadas en este texto enfatizan el papel fundamental de la interacción y de los procesos de comunicación en la constitución de los valores y creencias morales, resaltando también la importancia del análisis cualitativo e interpretativo de los procesos comunicativos para el estudio del desarrollo moral. Sin embrago, ellas difieren en relación a dos puntos.

El primero se refiere al papel activo, autónomo y creativo del sujeto en el proceso de internalización de los contenidos y lógicas relacionales socioculturales. Como colocamos anteriormente, los abordajes narrativos del constructivismo social no especifican el papel del individuo en el proceso de internalización, el cual aparece, a veces, como una mera apropiación o simple reproducción de las voces sociales combinadas de forma singular por el individuo; las perspectivas socioculturales resaltan la importancia del papel de la persona y su autonomía en los diferentes contextos organizados culturalmente.

$\mathrm{El}$ segundo punto se refiere a la forma como las diferentes perspectivas abordan la interpretación y el análisis de la narrativa del sujeto para el estudio del desarrollo y del funcionamiento moral. Como colocado por Rey (2002), el abordaje narrativo, en el marco del constructivismo social, disloca los conceptos relacionados con la constitución de lo intra-psíquico para el dominio público, comprendiendo los mismos como discursos socialmente construidos e ignorando, de esa forma, la dialéctica compleja entre lo social y lo individual en todos los dominios de la vida humana. Los abordajes narrativos del constructivismo social proponen un estudio del desarrollo y del funcionamiento moral a partir, única y exclusivamente, de la narrativa del sujeto en la resolución de problemas y dilemas morales, sin llevar en consideración la unidad inseparable entre acción y lenguaje.

Con relación al segundo punto podemos decir que para las perspectivas socioculturales decir y hacer constituyen una unidad de análisis inseparable. Como enfatizado anteriormente, la acción situada en contextos y prácticas socioculturales específicas y en constante diálogo con otras mentes activas es el punto más importante para el estudio del desarrollo, en el marco de una psicología de carácter sociocultural 
(Bruner, 1997; Valsiner \& Rosa, 2007). Siendo así, las perspectivas socioculturales abordan el desarrollo y funcionamiento moral por medio de narrativas contextualizadas en los diferentes sistemas de relaciones sociales y prácticas socioculturales, sin dejar de enfatizar el papel activo y creativo del sujeto. El desarrollo humano acontece a través de procesos complejos y está inmerso en una red de elementos de naturaleza semiótica (Rossetti-Ferreira, Amorim \& Silva, 2004). Sin embargo, ese desarrollo acontece en el marco de contextos y actividades socioculturales que pasan a tener un papel determinante (pero no determinista), dejando así de ser un trasfondo para tornarse un recurso o un instrumento de desarrollo.

Siendo así, el estudio y el análisis de los procesos de desarrollo debe considerar la persona en desarrollo como parte de un sistema, y buscar comprender las relaciones entre ella, las personas y los fenómenos de su entorno, así como los cambios que acontecen en el marco de esas relaciones. Eso implica, en nivel empírico, estudiar las transformaciones que acontecen en el sistema dinámico como resultado de muchas interacciones descentralizadas y locales que tienen lugar en un tiempo real (Rossetti-Ferreira, Amorim, Soares-Silva \& Oliveira, 2008).

Para abarcar el estudio de esas transformaciones, las perspectivas socioculturales combinan el análisis y la interpretación de las narrativas, con mayor o menor énfasis en el papel del afecto y de la emoción de los sujetos, con una multiplicidad de procedimientos metodológicos con el intuito de aprehender la complejidad dialéctica de los fenómenos socioculturales. Uno de los caminos promisorios para la comprensión de los cambios a lo largo del desarrollo y en el contexto de las relaciones e interacciones concretas ha sido el análisis microgenético, apuntado como una metodología propia para explorar el desarrollo de forma detallada (Valsiner, 2007; Rosseti-Ferreira et al., 2008).

\section{El análisis microgenético y el estudio de los procesos de desarrollo moral}

A partir de los aspectos abordados hasta el momento, esenciales para la comprensión de los procesos de desarrollo, destacamos aquí la importancia del análisis microgenético para el estudio del desarrollo moral. 
Desde el punto de vista de las perspectivas socioculturales y sus principales presupuestos teóricos, el estudio del desarrollo moral debe considerar cuatro aspectos fundamentales, pasibles de análisis a través de la microgénesis. En primer lugar la importancia de las interacciones sociales, ya que es por medio de la relación con los otros sujetos que el nińo elige los valores, reglas y normas que direccionarán su comportamiento moral. En segundo lugar, el papel de la cultura en el desarrollo humano, que asume relevancia central (Cole, 1992). En tercer lugar, es necesario considerar el papel crucial de la participación activa del sujeto en sus interacciones y en su proceso de desarrollo. Y en cuarto lugar, es fundamental comprender la cuestión mediante la integración sistémica, dinámica y compleja entre las prácticas culturales y las creencias y valores morales en el universo motivacional del individuo, que están en la base de su acción.

Los procesos de cambio representan un tema central en el estudio del desarrollo humano y, al mismo tiempo, constituyen un gran desafío para los investigadores, que deben llevar en cuenta numerosas cuestiones relativas al cambio. Lavelli, Pantoja, Hsu, Messinger y Fogel (2005) enfatizan cuestiones como: ¿Cuáles son los mecanismos y condiciones que pueden posibilitar procesos de cambio a nivel de desarrollo? ¿Cómo acontece el proceso de cambio? ¿Hasta qué punto los nuevos patrones de comportamiento tienden a suprimir o coexistir con los que ya existían? Cuestiones como esas se constituyen dificultades metodológicas fundamentales para los métodos tradicionales de investigación, que no se envuelven con la observación directa y detallada de los procesos de cambio cuando acontecen. Sin embargo, los desafíos consecuentes del estudio de los procesos de cambio van más allá de sus condiciones, y de los patrones de comportamiento que en ellos surgen y coexisten.

Por un lado, los procesos de desarrollo acontecen en el marco de interacciones, prácticas y contextos socioculturales específicos. El carácter complejo, dinámico e interrelacionado de esos dos elementos se constituye un desafío más para los estudiosos del desarrollo. $\mathrm{Al}$ mismo tiempo que es necesario el estudio minucioso de las transformaciones continuas y de las emergencias de novedades en las interacciones 
sociales (nivel micro), es fundamental considerar su interrelación con las prácticas y los contextos culturales (nivel macro). En relación a esta cuestión, Rogoff (2005) destaca el riesgo de aislar esos aspectos, concentrándose apenas en uno de ellos. Incluso cuando el estudio se centra en aspectos personales o interpersonales, las prácticas y los contextos culturales donde los mismos están inmersos deben permanecer en foco para una comprensión más amplia de lo que se pretende estudiar. En ese sentido, cabe al investigador desarrollar una visión sistémica del evento en estudio, enfocando las informaciones más importantes y manteniendo otras menos desatacadas, pero sin descartarlas. Según la autora, la totalidad del evento no puede ser estudiada de una vez, siendo que las distinciones entre lo que está en el primer plano y en el fondo residen en el análisis del investigador. Kelman y Branco (2004) también enfatizan la necesidad de una especie de 'zum' en el estudio de determinado proceso, con el intuito de captar los cambios significativos del desarrollo, que acontecen en un momento específico y en fracciones de segundo.

Por otro lado, los procesos de cambios significativos para el desarrollo se vehiculan principalmente a través de mensajes verbales y no verbales trasmitidos y re-significados por las personas en interacción. El estudio de esas especificidades comunicativas demanda un análisis muy detallado y pormenorizado, así como los recursos necesarios para el mismo. En ese sentido, Tacca (2000) señala la necesidad de recursos (grabaciones en vídeo) que permitan la observación de detalles y que favorezcan el análisis de la situación interactiva desde su inicio, destacando los procesos de construcción conjunta de significados, la comunicación y la metacomunicación entre las personas envueltas.

En el caso del desarrollo moral, la posibilidad de analizar los mensajes verbales y no verbales es de vital importancia, pues las creencias y los valores sociomorales son trasmitidos y re-significados a través de los dos tipos de mensajes, que pueden ser complementares, contradictorios o ambiguos. Tappan (1992) destaca la importancia de ter conciencia de la forma como los mensajes o 'voces morales' son comunicados a los niños, pues los mismos pueden transmitir y perpetuar normas culturales 
nocivas, estereotipadas e ideologías. Siendo así, los adultos deben tener conciencia del poder de sus voces y de cómo ellas influencian el funcionamiento moral de las próximas generaciones.

El carácter multifuncional de la comunicación, como apuntado por Fatigante, Fasulo y Pontecorvo (2004), tiene un papel fundamental en la trasmisión y re-significación de creencias y valores sociales. En función de eso, los tres niveles de abstracción presentes en la comunicación humana (denotativo, metalingüístico y metacomunicativo) deben ser contemplados, enfatizándose la comunicación relacional. Branco (2006) señala diferentes niveles de metacomunicación, entre los cuales destaca el nivel relacional como aquel de mayor importancia y significado para la psicología.

La metacomunicación relacional tiene como principal objetivo o función la construcción mutua de significados, por parte de los participantes, acerca de su propia interacción y/o relación. Siendo así, es una contribución relevante para la interpretación de los significados diversos que son construidos en conjunto en los procesos comunicativos. La función de la metacomunicación relacional es continua y puede acontecer tanto a través del lenguaje verbal como del lenguaje no verbal. Los participantes no solo usan signos lingüísticos, sino que también usan otros como gestos, el tono de la voz, posturas y movimientos corporales, ritmo del habla, entonación de la voz, miradas y expresiones faciales. Todos esos elementos tienen un papel preponderante en la atribución de sentido a la comunicación y, al mismo tiempo, son factores muy importantes para la mutua regulación de las acciones de los participantes (Fogel, 1993).

Los mensajes metacomunicativos están estrechamente relacionados con el tipo de actividad o con el contexto en que los participantes de la interacción se encuentran en un momento específico, y con los diferentes papeles que los participantes alternan durante la interacción, pasando particularmente por las relaciones de poder que se establecen y alternan durante el proceso interactivo. Los mensajes metacomunicativos también definen como los patrones comunicativos se ajustan a las orientaciones para objetivos de los participantes. Con eso, permiten que nuevas orientaciones para objetivos emerjan durante la interacción. 
El análisis microgenético ofrece al investigador del desarrollo recursos y procedimientos para enfrentar los desafíos anteriores, pues su objetivo básico es exponer la dinámica de los procesos de cambio, el origen (génesis) de los cambios que acontecen, así como el surgimiento de novedades en el curso del desarrollo. Es un análisis considerado 'micro' porque se orienta para detalles indíciales o recortes específicos de los eventos y contextos más amplios. Es genético porque busca relacionar esos recortes específicos de los eventos para detectar la emergencia de novedades y su relación con la totalidad de los eventos, y con otros planos de la cultura y de las prácticas socioculturales (Rossetti-Ferreira et al., 2008).

El análisis microgenético también posibilita un entendimiento amplio de las estrategias comunicativas y metacomunicativas usadas por los participantes de las situaciones de interacción. Específicamente, el análisis de todos las señales metacomunicativas facilita la comprensión de la intencionalidad de la acción de los sujetos, así como abre un espacio para entender la posible interpretación que los sujetos hacen de esas señales metacomunicativas, que orientan sus propias acciones e interacciones, y permiten la construcción conjunta de significados (Kelman \& Branco, 2004).

Desde un punto de vista teórico, el análisis microgenético permite interpretar la comunicación como un proceso dinámico, en el cual todos los participantes desempeńan un papel igualmente importante, resaltando la interdependencia entre los individuos y los diferentes contextos socioculturales. De forma general, podemos decir que, en el contexto de la microgénesis, la función central del lenguaje no es la expresión, sino la comunicación, en una perspectiva dialógica y en un flujo bidireccional, con las personas transformando y siendo transformadas a través de ella (Kelman \& Branco, 2004).

Además de las cuestiones anteriores, podemos señalar las múltiples funciones del análisis microgenético para el estudio del desarrollo dentro de ambientes como el contexto escolar. La microgénesis posibilita el análisis de la calidad de las interacciones sociales, y también posibilita detectar como esas interacciones facilitan o dificultan los procesos 
de aprendizaje (Branco \& Mettel, 1995; Branco \& Salomão, 2001; Kelman \& Branco, 2004). En estrecha relación con la calidad de las interacciones sociales, es posible estudiar las negociaciones que ocurren en el flujo interactivo entre profesor-niño y niño-niño.

La dinámica de esas negociaciones y las novedades que surgen a partir de ellas son de especial interés para el desarrollo moral. Las creencias y valores morales se vehiculan, de forma peculiar, en las negociaciones relacionadas con la resolución de conflictos interpersonales, y con el establecimiento y la modificación de las reglas sociomorales de los grupos. Por otro lado, la calidad de las interacciones sociales, que es un aspecto fundamental de la moralidad y que tiene en parte un carácter contractual (Piaget, 1932/1994), también está relacionada al compromiso con la dignidad de la condición humana, integrando las dimensiones psicológicas de la cognición, la emoción y la acción intencional, siempre presentes en la negociación con el otro.

En función de la complejidad de todas esas cuestiones, nos gustaría ilustrar los argumentos anteriores con el trecho de un análisis microgenético realizado en el curso de una investigación sobre desarrollo moral y prácticas pedagógicas en la Educación Infantil (Barrios, 2009). El estudio se fundamentó teórica y metodológicamente en una perspectiva sociocultural constructivista, y tuvo como objetivo identificar y analizar procesos, estrategias y mecanismos presentes en las interacciones profesora-nińos, que pudieran ser significativos para el desarrollo moral. Fueron grabadas en vídeo y analizadas microgeneticamente las interacciones de una profesora y sus 16 alumnos del $1^{\circ}$. Año de la Enseñanza Fundamental, de una escuela pública situada en Brasilia. Después de grabar las actividades cotidianas del grupo, realizamos una observación y un análisis previo de todas las sesiones grabadas en vídeo, con el objetivo de identificar los episodios más significativos para el estudio.

Los trechos seleccionados para el análisis están relacionados con el proceso de establecimiento y posible internalización de las reglas, con las estrategias de resolución de los conflictos interpersonales, y con la motivación social (ayuda, cooperación, agresión, hostilidad, 
individualismo, entre otros), que son aspectos resaltados por diversos autores (e.g. Branco, 1989; DeVries \& Zan, 1998; Turiel, 2002; Piaget, 1994) como significativos para el desarrollo de la moralidad. Los episodios seleccionados fueron transcritos, destacándose el tiempo de ocurrencia y la continuidad de las interacciones, las acciones y verbalizaciones de la profesora y de los niños, y las observaciones generales relativas a las actividades y al contexto en que las mismas acontecieron. Cada episodio fue dividido en unidades de análisis, constituidas por pequeños trechos de acciones y diálogos entre la profesora y los niños, lo que posibilitó un análisis minucioso de las interacciones. En el protocolo del Anexo 1, presentamos el análisis microgenético de una situación de conflicto entre dos niños del grupo. El episodio fue titulado "Pero yo pagué con el dinero que tenía", a partir del enunciado de uno de los niños envueltos en la situación de conflicto.

El análisis del protocolo del Anexo 1 permitió identificar cuestiones fundamentales para el desarrollo de la moralidad. En primer lugar, los valores y creencias sociomorales que se vehicularon en el contexto de esas interacciones especificas, y como los mismos se insirieron en posibles procesos de cambio o énfasis en función de la dinámica relacional. Si al principio del episodio los niños presentaron una solución para el conflicto de acuerdo con sus creencias, al final del mismo ellos buscaron otras soluciones, una vez que su estrategia de reparación se mostró inadecuada de acuerdo con las creencias y valores colocados por la profesora. En segundo lugar, el papel de la metacomunicación relacional en la construcción mutua de significados por parte de los participantes. A lo largo del episodio, la inadecuación de la estrategia de reparación fue metacomunicada por la profesora a través del tono de la voz, que fue de reprobación y dejó claro su posicionamiento afectivo con relación a la situación de conflicto.

También podemos decir que la profesora se colocó de forma autoritaria, direccionando la discusión y determinando el tiempo de duración de la misma. En tercer lugar, el análisis minucioso de las acciones y de la comunicación posibilitó detectar como las mismas pueden facilitar o dificultar los procesos de aprendizaje y desarrollo. A lo largo del episodio, 
la profesora escuchó parcialmente a los niños y no avanzó en la discusión moral, en función de su objetivo principal que era la escritura de la palabra Pascua. Como colocamos anteriormente, la actividad con contenidos escolares acabó prevaleciendo en relación a la discusión sobre cuestiones sociomorales y la situación no fue aprovechada como espacio de aprendizaje y desarrollo de la moralidad y de los procesos de socialización. Al final del episodio, la profesora encierra la discusión sin ofrecer otras alternativas de resolución para el conflicto, e indicando que discutir y aprender a resolver los conflictos interpersonales puede ser importante, pero no tan importante como la actividad de escritura.

El análisis microgenético de la dinámica relacional en esa situación de conflicto real, no solo permitió estudiar los valores sociomorales compartidos y negociados por ese grupo determinado, sino que también permitió conocer cuál es el espacio y la importancia del desarrollo moral en ese contexto escolar y en esas prácticas escolares mediadas por interlocuciones entre la profesora y el grupo de alumnos. Como colocamos al principio de este texto, el estudio del desarrollo moral debe buscar tanto la comprensión científica del fenómeno y su importancia para el desarrollo integral del ser humano, como su papel en el sistema educacional, pues es en ese contexto que los niños y los jóvenes expanden sus posibilidades de socialización más allá de la familia y de la comunidad, construyendo las bases mediadas en el cotidiano para la identificación sociomoral.

\section{Referencias}

Araújo, U. F. de (1996). O ambiente escolar e o desenvolvimento do juízo moral infantil. En L. de Macedo (Org.), Cinco estudos de educação moral (pp. 105-135). São Paulo: Casa do Psicólogo.

Ardilla-Rey, A. \& Killen, M. (2001). Middle class Colombian children's evaluations of personal, moral, and social-conventional interactions in the classroom. International Journal of Behavioral Development, 25(3), 246-255. 
Bakhtin, M. M. (1981). Problemas da poética de Dostoiesvky. São Paulo: Hucitec.

Bakhtin, M. M. (1993). Toward a philosophy of the act. En V. Liapunov \& M. Holquist (Eds.), Toward a philosophy of the act (pp. XXIV-106). Austin: University of Texas.

Barrios, A. (2009). Desenvolvimento Moral e Práticas Pedagógicas na Educação Infantil: Um Estudo Sociocultural Construtivista. $246 \mathrm{f}$. Dissertação de Mestrado, Instituto de Psicologia da Universidade de Brasília.

Bauman, Z. (2001). Postmodern ethics. En S. Seidman \& J. C. Alexander (Orgs.), The new social theory reader (pp. 138-145). Londres: Routledge.

Biaggio, A. M. B. (1983). Desenvolvimento de valores: Um estudo piloto. Educação e Realidade, 8(1), 25-33.

Branco, A. U. (2006). Crenças e práticas culturais: Co-construção e ontogênese de valores sociais. Revista Pro-Posiçóes (UNICAMP), 17, 2(50), 139-155.

Branco, A. U. (1989). Socialização na pré-escola: O papel da professora $e$ da organização das atividades no desenvolvimento de interaçóes sociais entre crianças. $280 \mathrm{f}$. Tese de Doutorado, Instituto de Psicologia da Universidade de Sáo Paulo.

Branco, A. U. \& Mettel, T. (1995). O processo de canalização cultural das interaçóes criança-criança na escola. Psicologia: teoria e pesquisa, 11(1), 13-22.

Branco, A. U. \& Salomão, S. (2001). Cooperação, competição e individualismo: Pesquisa e contemporaneidade. Temas em Psicologia da SBP, 9(1), 11-18.

Bruner, J. (1997). Atos de significação. Porto Alegre: Artes Médicas.

Canclini, N. G. (2003). Cultura híbrida. São Paulo: Edusp.

Cole, M. (1992). Culture in development. En M. H. Bornstein \& M. E. Lamb (Orgs.), Developmental psychology: An advanced textbook (pp. 731-788). Hillsdale, NJ: Lawrence Earlbaum Associates. 
Costa, E. V. \& Lyra, M. C. D. P. (2002). Como a mente se torna social para Barbara Rogoff? A questão da centralidade do sujeito. Psicologia: Reflexão e Crítica, 15(3).

DeVries, R. \& Zan, B. (1998). A ética na educação infantil: O ambiente sócio-moral na escola. Porto Alegre: Artes Médicas.

Duska, R. \& Whelan, M. (1994). O desenvolvimento moral na idade evolutiva: Um guia de Piaget a Kohlberg. São Paulo: Loyola.

Emerson, C. (1984). Editor's preface. En M. M. Bakthin, Problems of Dostoievsky's poetics (pp. XXIX-XLIII). Mineapolis: University of Minesota Press.

Fatigante, M., Fasulo, A. \& Pontecorvo, C. (2004). This is not a dinner: Metacommunication in family dinnertime conversations. En A. U. Branco \& J. Valsiner (Eds.), Communication and metacommunication in human development (pp. 33-81). Greenwood, CT: Information Age Publishers.

Fidalgo, Z. (2004). Psicologia Cultural e Desenvolvimento Humano: Um encontro com Barbara Rogoff. Análise Psicológica, 22(1).

Fogel, A. (1993). Developing through relationships. Londres: Harvest Wheatsheaf/ University of Chicago Press.

Gilligan, C. (1982). Uma voz diferente. Rio de Janeiro: Rosa dos tempos.

Goodnow, J. (1995). Parenting and the transmission and internalization of values. En J. E. Grusec \& L. Kuczynski (Eds.), Parenting and children's internalization of values (pp. 333-397). Nueva York: John Wiley \& Sons.

Kelman, C. A. \& Branco, A. U. (2004). Análise microgenética em pesquisa com alunos surdos. Revista Brasileira de Educação Especial Marilia, 10(1), 93-106.

Kohlberg, L. (1984). Essays on moral development. Vol. 2: The psychology of moral development: moral stages, their nature and validation. Nueva York: Harper \& Row.

Kurtines, W. \& Pimm, J. (1983). The moral development scale: A Piagetian measure of moral judgment. Educational and Psychological Measurement, 43, 89-105. 
La Taille, Y. de (1998). Limites: Três dimensóes educacionais. São Paulo: Ática.

La Taille, Y. de (2001). Desenvolvimento moral: A polidez segundo as crianças. Cadernos de Pesquisa, 114, 89-119.

La Taille, Y. de (2006). Moral e ética: Dimensóes intelectuais e afetivas. Porto Alegre: Artmed.

Lavelli, M., Pantoja, A. P. F., Hsu, H., Messinger, D. \& Fogel, A. (2005). Using microgenetic desings to study change processes. En D. M. Teti (Org.), Handbook of research methods in developmental science (pp. 40-65). Malden: Blackwell Publishing.

Lourenço, O. M. (1998). Psicologia de desenvolvimento moral: Teoria, dados e implicaçóes. Coimbra, Portugal: Livraria Almeida.

Lourenço. O. \& Kahn, P. (2000) Raciocínio ecológico-moral: Um estudo desenvolvimentista numa amostra de sujeitos de Lisboa. Análise Psicológica, 4(18), 425-435.

Lustosa, A. V. (2005). A compreensão da moralidade: Contribuiçôes teóricas da psicologia do desenvolvimento. En M. A. Dessen \& A. L. Costa Jr. (Orgs.), A ciência do desenvolvimento humano: tendências atuais e perspectivas futuras (pp. 249-263). Porto Alegre: Artmed.

Martins, R. A. (1995). Concepçóes sobre regras morais e convencionais em crianças de pré-escola e do primeiro grau. Psicologia: Teoria e Pesquisa, 11, 3, 203-211.

Martins, L. C. (2000). Crenças e valores morais em pré-adolescentes: Uma análise sociocultural construtivista de processos comunicativos em contextos semi-estruturados. $223 \mathrm{f}$. Tese de Doutorado, Instituto de Psicologia da Universidade de Brasília, Brasília.

Martins, L. C \& Branco, A. U. (2001). Desenvolvimento moral: Consideraçóes teóricas a partir de uma abordagem sociocultural construtivista. Psicologia: Teoria e Pesquisa, 17(2), 169-176.

Packer, M. J. \& Richardson, E. (1991). Analytic hermeneutics and the study of morality in action. En W. M. Kurtines \& J. L. Gewirtz (Orgs.), Handbook of moral behavior and development. Vol. 1: Theory (pp 335-371). New Jersey: Lawrence Erlbaum Associates. 
Piaget, J. (1994). O juizo moral na criança. São Paulo: Summus. (Originalmente publicado en 1932).

Puig, J. M. (1998). A construção da personalidade moral. São Paulo: Ática. Rest, J. (1984). The major components of morality. En W. Kurtines \& J. Gewirtz (Eds.), Morality, moral behavior, and moral development (pp. 24-40). Nueva York: Wiley.

Rey, F. G. \& Martínez, A. M. (1989). La personalidad, su educación y desarrollo. La Habana: Pueblo y Educación.

Rey, F. G. (2002). Pesquisa qualitativa em psicologia: Caminhos e desafios. Sao Paulo: Thomson.

Rogoff, B. (2005). A natureza cultural do desenvolvimento bumano. Porto Alegre: Artmed.

Rossetti-Ferreira, M. C., Amorin, K. S. \& Silva, A. P. S. (2004). Rede de significaçóes: Alguns conceitos básicos. En M. C. RossettiFerreira, K. S. Amorin, A. P. S. Silva \& A. M. A. Carvalho (Orgs.). Rede de significaçóes: $E$ o estudo do desenvolvimento humano (pp. 23-33). Porto Alegre: Artmed.

Rossetti-Ferreira, M. C.; Amorim, K. S.; Soares-Silva, A. P. \& Oliveira, Z. M. R. (2008). Desafios metodológicos na perspectiva da rede de significaçóes. Cuadernos de Pesquisa, 38(133), 147-170.

Selman, R. (1980). The growth of interpersonal understanding. Nueva York: Academic Press.

Shweder, R. A. \& Much, N. C. (1987). Determinations of meaning: Discourse and moral socialization. En W. M. Kurtines \& J. L. Gewirtz (Eds.), Moral development through social interaction (pp. 197-244). Nueva York: Wiley.

Tacca, M. C. (2000). Ensinar e aprender: A construção de significados na interação professor-alunos em atividades estruturadas. $257 \mathrm{f}$. Tese de Doutorado, Instituto de Psicologia da Universidade de Brasília, Brasília.

Tappan, M. B. (1992). Texts and contexts: Language, culture, and the development of moral functioning. En L. T. Winegar \& J. Valsiner (Eds). Children's development within social context (Vol. 1, pp. 93-117). Hillsdale, NJ: Lawrence Earlbaum Associates. 
Turiel, E. (2002). The culture of morality: Social development, context and conflict. Cambridge: Cambridge University Press.

Valsiner, J. (2007). Culture in minds and societies: Foundations of cultural psychology. Nueva Delhi: Sage.

Valsiner, J. \& Rosa, A. (2007). The Cambridge handbook os sociocultural psychology. Cambridge:Cambridge University Press.

Volosinov, V. N. (1995). Marxismo e filosofia da linguagem. (Trads. Michael Lahud e Yara Frateschi Vieira). São Paulo: Hucitec. (Originalmente publicado en 1929).

Vygotsky, L. S. (1987). Historia del desarrollo de las funciones psíquicas superiores. La Habana: Científico-Técnica. (Originalmente publicado en 1960).

Vygotsky, L. S. (1968). Pensamiento y lenguaje. La Habana: Instituto del Libro. (Originalmente publicado en 1934).

Wertsch, J. \& Smolka, A. L. B. (2003). Continuando o diálogo: Vygotsky, Bakhtin e Lotman. En H. Daniels (Org.), Vygotsky em foco: Pressupostos e desdobramentos (pp. 121-150). São Paulo: Papirus.

Recibido: 05 de enero de 2012 Aceptado: 21 de octubre de 2012 


\section{Anexo 1}

\section{Protocolo - Análisis Microgenético del Episodio "Pero yo pagué con el dinero que tenía"}

ACTIVIDAD: Rueda de conversación. Los niños están sentados en el piso, en rueda. La profesora está de pie, al lado de la rueda, con el material de las primeras actividades en la mano. Los niños y la profesora conversan sobre una actividad que hicieron el día anterior, para encerrar las clases. La actividad consistió en pintar la cáscara de un huevo cocido, como símbolo de la Pascua. Durante la actividad, la profesora explicó y conversó con los niños sobre el significado de la Pascua. Al final del día los niños se llevaron el huevo pintado para la casa. Los nińos están atentos a la conversación de la rueda y participan respondiendo a las preguntas de la profesora.

\section{LEYENDA:}

P3: Profesora del grupo.

M: Nińos, enumerados de acuerdo con su posición en la rueda.

F: Niñas, enumeradas de acuerdo con su posición en la rueda.

$\rightarrow$ Inicio del flujo de las interacciones.

TIEMPO: $7 \mathrm{~h} 58$

\begin{tabular}{|l|l|}
\hline PROFESORA - P3 & NIÑOS \\
\hline Unidad de Análisis 2 - 00:06 - 8h04 & $\begin{array}{l}\rightarrow \text { M6: ¡Tía! ¡Tía! (Con el brazo } \\
\text { levantado, solicitando tiempo para } \\
\text { hablar.) }\end{array}$ \\
$\begin{array}{l}\text { La profesora mira a M6. } \\
\text { P3: Ahora, M6. Yo me puse muy triste } \\
\text { con M6, porque alguien, allá fuera... } \\
\text { Yo lo oí desde aquí, que yo salí de aquí } \\
\text { a las dos, o dos y media. Hubo alguien } \\
\text { que rompió el huevo de M6. }\end{array}$ & $\begin{array}{l}\text { M6: Yo lo sé. Fue M9. (Apuntando para } \\
\text { M9.) }\end{array}$ \\
$\begin{array}{l}\text { P3: ¡Eso mismo! Me puse triste con lo } \\
\text { que pasó. }\end{array}$ & M9: Yo no sabía que lo estaba \\
$\begin{array}{l}\text { P3: ¡Él cuidando tanto el huevo! ¡Fue } \\
\text { allá y le rompió el huevo! (Mirando } \\
\text { fijamente para M9.) }\end{array}$ & rompiendo. \\
\hline
\end{tabular}


En el trecho 2, el grupo participa en una discusión sobre un conflicto entre dos niños. Es importante resaltar que el niño perjudicado durante el conflicto quiere hablar y discutir sobre lo que pasó. Sin embargo, fue la profesora la que tomó la palabra y expuso el conflicto para el grupo, centralizando la discusión en torno de ella. La profesora se coloca de forma afectiva en relación a la situación ("Yo me puse muy triste con M6...”) y metacomunica, a través del tono de la voz, su desagrado en relación a las acciones de $\mathrm{M} 9$.

M9 señala que la situación fue sin querer, lo que no es aprovechado por la profesora para esclarecer los acontecimientos y ampliar el marco de la discusión. La profesora adopta una postura acusadora (“¡Él cuidando tanto el huevo! ¡Fue allá y le rompió el huevo!”), sin saber lo que había acontecido exactamente.

\section{Unidad de Análisis 3 - 00:07 - 8h05 $\rightarrow$ M9: Pero yo lo pagué.}

P3: ¿Cómo fue que lo pagaste?

P3: ¡Con dinero! ¡Dios mío!

\section{M9: Con el dinero que yo tenía.}

M6 mira para la profesora.

M5: Él lo pidió. (Apuntando para M6.) M6: Sí.

P3: Pero el problema no era el dinero. ¿No era que tú lo habías hecho con cariño? (Mirando a M6.)

M6 hace un gesto de afirmación con la cabeza.

En este trecho, los niños explican la estrategia de reparación que usaron para negociar y resolver el conflicto. Frente a la estrategia, la profesora comunica de forma explícita su desaprobación y trasmite sus creencias y valores: cuando las cosas tienen valor afectivo no se deben reparar a través del dinero. Sin embargo, no sugiere lo que los niños pueden hacer en ese caso.

Aunque la estrategia de reparación no sea adecuada de acuerdo con las creencias y valores colocados por la profesora, es importante resaltar dos cuestiones significativas para el desarrollo moral. Los nińos intentaron resolver el conflicto entre ellos de una forma autónoma, y percibieron la necesidad e importancia de una reparación en ese contexto. Esas dos cuestiones no fueron percibidas, ni abordadas por la profesora durante la discusión sobre el conflicto. 


\section{Unidad de Análisis 4 - 00:08 - 8h06 \\ P3: ¿Bueno, y cómo quedó?}

P3: ¡No, no podía romper el huevo de él! ¡Eso no es correcto! ¡i Mira que idea!! ¡iMira que idea esa!! ¿¡Podía romper el huevo!? ¡iNo!!

La profesora mira para M6.

P3: Bueno... ¿Cómo fue que tú hiciste? ¿Tú llegaste a la casa y cómo hiciste? ¿Se rompió mucho?

P3: ¡¿Mucho?!

P3: ¡Ah bueno! ¡Sólo se rajó! Entonces no fue mucho. ¡Se pudo resolver! Está bien entonces. Vamos a continuar. Ayer ustedes vieron la palabra Pascua y estudiamos lo que es la Pascua. Hoy, vamos a escribir la palabra Pascua, vamos a ver con que letra empieza. Vamos a ver quién se va a llevar la cajita. Hoy vamos ir al parque, porque ayer no llovió. ¿Está bien? También vamos a usar los juguetes pedagógicos. La profesora se para frente a la rueda, con un ábaco en la mano.

P3: Vamos a ver cuántas niñas vinieron hoy.
M12: ¡Bueno, el otro podía romper el huevo de él! (Señalando la posibilidad de M6 romper el huevo de M9 y pagar con la misma moneda.)

M12 se queda en silencio mirando a la profesora.

M6 hace un gesto afirmativo con la cabeza.

M6 hace un gesto afirmativo con la cabeza.

M9: ¡Sólo lo rajé!

Los niños observan y atienden lo que la profesora dice. Todos están en silencio.

F1: Dos. 
En el trecho 4, la discusión sobre el conflicto continúa. Una vez que la estrategia de resolución de los niños fue inadecuada, otro niño del grupo (M12) sugiere pagar con la misma moneda, rompiendo el huevo de M9. La profesora interfiere comunicando e metacomunicando su espanto y desaprobación en relación a esa idea, sin embargo, no avanza en la discusión ni busca junto con los niños otras estrategias de resolución. En ese contexto, el conflicto se queda sin una solución adecuada.

A pesar de la motivación y el interés de los niños por la discusión, la profesora encierra la misma en función del objetivo que era utilizar el trabajo del día anterior para escribir la palabra Pascua. Siendo así, la actividad con contenidos escolares acaba prevaleciendo en relación a la discusión sobre cuestiones sociomorales. El discurso de la profesora, en ese encerramiento, se muestra contradictorio en relación al discurso anterior: como sólo se rajó, entonces la cuestión no es tan importante y podemos continuar. 